ERRATUM

doi:10.1038/nature12067

\title{
Erratum: A sub-Mercury-sized exoplanet
}

Thomas Barclay, Jason F. Rowe, Jack J. Lissauer, Daniel Huber, François Fressin, Steve B. Howell, Stephen T. Bryson, William J. Chaplin, Jean-Michel Désert, Eric D. Lopez, Geoffrey W. Marcy, Fergal Mullally, Darin Ragozzine, Guillermo Torres, Elisabeth R. Adams, Eric Agol, David Barrado, Sarbani Basu, Timothy R. Bedding, Lars A. Buchhave, David Charbonneau, Jessie L. Christiansen, Jørgen Christensen-Dalsgaard, David Ciardi, William D. Cochran, Andrea K. Dupree, Yvonne Elsworth, Mark Everett, Debra A. Fischer, Eric B. Ford, Jonathan J. Fortney, John C. Geary, Michael R. Haas, Rasmus Handberg, Saskia Hekker, Christopher E. Henze, Elliott Horch, Andrew W. Howard, Roger C. Hunter, Howard Isaacson, Jon M. Jenkins, Christoffer Karoff, Steven D. Kawaler, Hans Kjeldsen, Todd C. Klaus, David W. Latham, Jie Li, Jorge Lillo-Box, Mikkel N. Lund, Mia Lundkvist, Travis S. Metcalfe, Andrea Miglio, Robert L. Morris, Elisa V. Quintana, Dennis Stello, Jeffrey C. Smith, Martin Still \& Susan E. Thompson

Nature 494, 452-454 (2013); doi:10.1038/nature11914

In this Letter, the Centro de Astrobiologia affiliation (number 11, associated with authors David Barrado and Jorge Lillo-Box) was listed incorrectly; the correct address is: 'Departamento Astrofísica, Centro de Astrobiología (INTA-CSIC), ESAC campus, PO Box 78, E-28691 Villanueva de la Cañada, Spain'. This has been corrected in the HTML and PDF versions of the manuscript. 